\title{
Mortality in Patients with Type 2 Diabetes: Impact of Age, Glycemic Control, and Renal Complications
}

\section{Leonardo Roever $^{1 *}$ and Anaisa Silva Roerver Borges ${ }^{2}$}

${ }^{1}$ Department of Clinical Research, Federal University of Uberlandia, Uberlandia, Brazil ${ }^{2}$ Master Institute of Education President Antonio Carlos - IMEPAC-Araguari, Brazil

\section{Introduction}

Diabetes mellitus is a chronic degenerative metabolic disease with worldwide prevalence approaching 400 million people. In patients with type 2 diabetes, hypertension, metabolic syndrome, low circulating testosterone levels, isolated fasting hyperglycemia and obesity paradox is associated with a range of adverse outcomes including cardiovascular disease and premature mortality [1-7].

Tancredi and colleagues assessed these risks according to glycemic control and renal complications among persons with type 2 diabetes. The authors included patients with type 2 diabetes who were registered in the Swedish National Diabetes Register. For each patient, five controls were randomly selected from the general population and matched according to age, sex, and county. The mean follow-up was 4.6 years in the diabetes group and 4.8 years in the control group. Overall, 77,117 of 435,369 patients with diabetes (17.7\%) died, as compared with 306,097 of 2,117,483 controls (14.5\%) (Adjusted hazard ratio (HR), 1.15; 95\% confidence interval [CI], 1.14 to 1.16). The rate of cardiovascular death was $7.9 \%$ among patients versus $6.1 \%$ among controls (adjusted HR, 1.14 ; $95 \%$ CI, 1.13 to 1.15 ). The excess risks of death from any cause and cardiovascular death increased with younger age, worse glycemic control, and greater severity of renal complications. When compared with controls, the HR for death from any cause among patients younger than 55 years of age who had a glycated hemoglobin level of $6.9 \%$ or less ( $\leq 52 \mathrm{mmol}$ per mole of nonglycated hemoglobin) was 1.92 (95\% CI, 1.75 to 2.11); the corresponding HR among patients 75 years of age or older was 0.95 (95\% CI, 0.94 to 0.96 ). Among patients with normoalbuminuria, the hazard ratio for death among those younger than 55 years of age with a glycated hemoglobin level of $6.9 \%$ or less, as compared with controls, was 1.60 (95\% CI, 1.40 to 1.82 ); the corresponding HR among patients 75 years of age or older was 0.76 ( $95 \%$ CI, 0.75 to 0.78 ), and patients 65 to 74 years of age also had a significantly lower risk of death (HR, $0.87 ; 95 \% \mathrm{CI}, 0.84$ to 0.91 ) [8].
Mortality among persons with type 2 diabetes depending on age, glycemic control, and renal complications. A large cohort study should be conducted to definitively determine the clinical significance of these factors, its correlation with mortality. Potential treatments such as lifestyle modification, ectopic fat reduction, surgery and medications should be investigated.

\section{References}

1. Jansson SP, Andersson DK, Svardsudd K (2015) Mortality and cardiovascular disease outcomes among 740 patients with new-onset Type 2 diabetes detected by screening or clinically diagnosed in general practice. Diabet Med.

2. Marcelino-Rodríguez I, Elosua R, Pérez MD, Fernández-Berges D, Guembe MJ, et al. (2015) On the problem of type 2 diabetes-related mortality in the Canary Islands, Spain, The DARIOS Study. Diabetes Res Clin Pract.

3. Liu JJ, Lim SC, Yeoh LY, Su C, Tai BC, et al. (2015) Ethnic disparities in risk of cardiovascular disease, end-stage renal disease and all-cause mortality: a prospective study among Asian people with Type 2 diabetes. Diabet Med.

4. Tint A, Hoermann R, Wong H, Ekinci El, Maclsaac RJ, et al. (2015) Association of Sex Hormone Binding Globulin and Free Testosterone with Mortality in Men withType 2 Diabetes Mellitus. Eur J Endocrinol 9: 15-0672.

5. Won KB, Hur SH, Cho YK, Yoon HJ, Nam CW, et al. (2015) Comparison of 2-year mortality according to obesity in stabilized patients with type 2 diabetes mellitus after acute myocardial infarction: results from the DIAMOND prospective cohort registry. Cardiovasc Diabetol 14: 141.

6. Mirbolouk M, Hajebrahimi MA, Akbarpour S, Tohidi M, Azizi F (2015) Different glucose tolerance status and incident cardiovascular disease and all-cause mortalityamong elderly Iranians. Geriatr Gerontol Int

7. Nathan DM (2015) Diabetes: Advances in Diagnosis and Treatment. JAMA 8-314: 1052-1062.

8. Tancredi M, Rosengren A, Svensson AN, Kosiborod M, Pivodic A (2015) et al. Excess Mortality among Persons with Type 2 Diabetes. N Engl J Med $373: 1720-1732$

Received November 10, 2015; Accepted December 07, 2015; Published December 30, 2015

Citation: Roever L, Borges ASR (2015) Mortality in Patients with Type 2 Diabetes: Impact of Age, Glycemic Control, and Renal Complications. J Diabetes Metab 7: e119. doi:10.4172/2155-6156.1000e119

Copyright: $\odot 2015$ Roever L, et al. This is an open-access article distributed under the terms of the Creative Commons Attribution License, which permits unrestricted use, distribution, and reproduction in any medium, provided the original author and source are credited. 